\title{
LA QUÍMICA Y LA LENGUA ESPAÑOLA EN EL S. XIX ${ }^{1}$
}

\section{Cecilio Garriga}

Universitat Autònoma de Barcelona

\section{RESUMEN}

El léxico de la química registra un cambio importante a partir del último cuarto del siglo XVIII. La traducción de la Nueva nomenclatura química conlleva la llegada de los nuevos términos al español. En este estudio se explican y ejemplifican las tensiones que la llegada de nuevos términos provoca en el sistema léxico. Todo ello teniendo en cuenta los últimos datos proporcionados desde la historia de la ciencia.

PALABRAS CLAVE: español, química, diccionario, lenguas de especialidad, léxico.

\section{SUMMARY}

The lexis of chemistry registered an important change from the final quarter of the C.18. The translation of the Nueva nomenclatura química («New Chemical Nomenclature») brought in its wake the arrival of new terms to the Spanish language. Bearing in mind the latest data provided from the history of science, this study explains and exemplifies the tension within the lexical system created by the arrival of the new terms.

KEY WORDS: spanish, chemistry, dictionary, languages for specific purpose, vocabulary.

\section{INTRODUCCIÓN}

No parece necesario insistir aquí en la importancia de la historia de la ciencia para los estudios lingüísticos que centren su análisis en la lengua de la ciencia y de la técnica, ni en la poca atención que se ha prestado hasta ahora a este ámbito por parte de la filología española, ni en el desinterés tradicional

1 Este estudio se enmarca en el proyecto de investigación Catálogo de neologismos del léxico científico y técnico del s. XIX, financiado parcialmente por el MCYT (BFF2001-2478). 
hacia la lengua del siglo XIX ${ }^{2}$. Las posturas ideológicas que alimentaron la llamada «polémica de la ciencia española ${ }^{3}$ y que impedían aclarar la realidad histórica, han tenido también su reflejo en el ámbito de la filología 4 .

Para superar este enfoque, en este estudio se trata precisamente la lengua de una de las ciencias más características, la química, que puede servir como modelo para el análisis de otras lenguas de especialidad por varias razones: por su proceso de institucionalización durante el siglo XIX, por la importancia de las discusiones lingüísticas que tienen lugar entorno de esta ciencia ${ }^{5}$, porque combina una nueva nomenclatura con términos tradicionales, y por tratarse de un léxico importado y adaptado por el español desde otras lenguas, fundamentalmente el francés.

En su planteamiento trataré paralelamente del estado y desarrollo de la química desde el punto de vista de la historia de la ciencia, de las actitudes lingüísticas ante el desarrollo de la lengua científica y técnica y de los instrumentos al alcance del filólogo para su estudio en el siglo XIX. Después mostraré algunos ejemplos de cómo el nuevo vocabulario va penetrando en el español, y de las tensiones lingüísticas que esta situación genera.

\section{Historia DE LA CIENCIA E HISTORIA DE LA LENGUA}

Cabe decir, en este sentido, que el punto de partida de la química al inicio del siglo XIX era bastante modesto, ya que hasta ese momento no había dispuesto de un amplio cuerpo de conocimientos compartidos, ni de una indus-

2 Véase, a este respecto, GutiÉrrez CUADRAdo, J. (2001), «Lengua y ciencia en el siglo XIX español, el ejemplo de la química», en BARgalló, M.; Forgas, E.; GARriga, C.; RuBIO, A. y SCHNitzer, J. (eds.), Las lenguas de especialidad y su didáctica, Tarragona, Universitat Rovira i Virgili, 181-196, p. 181-182.

3 LÓPEZ PiÑERO, J. L. (1992), «Introducción», en LÓPEZ PIÑERO, J. L. (ed.): La ciencia en la España del siglo XIX, Madrid, Marcial Pons, 11-18, p. 11.

4 Haciendo uso de este tópico, Lázaro Carreter afirmaba que «la falta de léxico técnico y científico es un mal que arrastra nuestro idioma desde el Renacimiento hasta nuestros días». En LÁzAro CARreter, F. (1985), Las ideas lingüisticas en España durante el siglo XVIII, Barcelona, Crítica, p. 284.

5 El contenido lingüístico inherente a la química, reconocido desde la propia historia de la ciencia, se pone de manifiesto en múltiples estudios, entre los que cabe destacar, por su envergadura, el ya clásico de CROSLAND, M. P. (1962), Historical Studies in the Language of Chemistry, London, Heinemann Educational Books. Y, para el español, el de GARCÍA BELMAR, A. y BERTOMEU SÁNCHEZ, J. R. (1999), Nombrar la materia. Una introducción histórica a la terminología química, Barcelona, Serbal. 
tria demasiado importante que aprovechara los avances de la ciencia ${ }^{6}$. Sin embargo, esta situación estaba empezando a cambiar, ya que la propuesta de una nueva nomenclatura para la química inorgánica por parte de Lavoisier y sus colaboradores, y las aplicaciones al tratamiento de los tejidos, a la minería o a la fabricación de pólvoras, estaban dando a esta ciencia un mayor protagonismo. Durante el siglo XIX el desarrollo fue espectacular, hasta el punto de llegar a calificarlo como «el siglo de la química», sobre todo debido a los avances obtenidos en la química orgánica y en la clasificación de los elementos químicos a través del sistema periódico.

Pero el desarrollo de la química en España no fue uniforme con respecto a los avances europeos. Los últimos años del siglo XVIII habían sido realmente florecientes, si no en grandes logros, sí en la penetración de las últimas propuestas de los químicos franceses. La Corona se interesa por los resultados aplicables con fines militares y económicos, y protege y promociona el cultivo de esta ciencia ${ }^{7}$. Su política de contratación de químicos extranjeros para que enseñaran y ejercieran en España da los frutos esperados ${ }^{8}$. A la vez, se envía a científicos pensionados para que se formen en los laboratorios de los centros más activos del continente, entre los que destacan Aréjula y Carbonel19. El resultado es una nómina de químicos españoles que están al día de

6 Portela, E. (1998), La química en el siglo XIX, Madrid, Akal, p. 7-12.

7 Véase, a este respecto, el estudio de PESET, J. L. y LAfUente, A. (1988), «Las actividades e instituciones científicas en las España ilustrada», en SELlÉS, M.; PESET, J. L. y LAFUENTE, A. (eds.), Carlos III y la ciencia de la Ilustración, Madrid, Alianza, 29-79.

8 En Portela, E. (1999), La química ilustrada, Madrid, Akal, p. 48, se destacan los casos de L. J. Proust y F. Chavaneau que llegan para dirigir la cátedra de química de Vergara, C. Storr y J. M. Hoppensack para dirigir las minas de Almadén, etc. Véase este mismo proceso en GaGO, R. (1988a), "The New Chemistry in Spain», Osiris, 4, 169-192. Una visión general en el marco de las ciencias, en SARRAILH, J. (1985), La España Ilustrada de la segunda mitad del siglo XVIII, Madrid, FCE. Sobre la cátedra de química de Vergara, véase GAGO, R. (1978), «Bicentenario de la fundación de la Cátedra Química de Vergara. El proceso de constitución», Llull, 2, 5-18; y GAGO, R. y Pellón, I. (1994), Historia de las Cátedras de Química y Mineralogía de Bergara a finales del siglo XVIII. Bergara, Ayuntamiento. Sobre Proust, véase GAgO, R. (1990), «Luis Proust y la cátedra de química de la Academia de Artillería de Segovia», en Proust, L., Anales del Real Laboratorio de Química de Segovia, Segovia, A. Espinosa [1795]; facsímil en Segovia, Academia de Artillería, 5-51.

9 Los dos casos más representativos son Carbonell, que va a estudiar a Montpellier con Chaptal, y Aréjula que estudia en París con Fourcroy. Sobre Carbonell véase NIETO, A. (1996), «Martí i Franquès, Carbonell i Bravo, i els usos de la nova química a la Catalunya il.lustrada», en IZQUIERDO, M. et alii (eds.), Lavoisier $i$ els origens de la química moderna, 200 anys després (1794-1994), Barcelona, SCHCYT, 159-184; y sobre Aréjula, GAGO, R. y 
las más recientes novedades, ya que a los dos ya mencionados, hay que añadir nombres como los de Gutiérrez Bueno, Munárriz, Martí i Franquès, García Fernández, los hermanos Elhuyar, Garriga y Buach, Sancristóbal... ${ }^{10}$. Como fruto de este proceso, se traducen al español las principales obras de los autores franceses ${ }^{11}$, lo que motiva la llegada al español de las propuestas léxicas que designan no solo los nuevos elementos químicos y sus combinaciones, sino los nuevos conceptos, operaciones, utensilios, etc... El estudio de estos textos permite describir con detalle el proceso de incorporación de voces químicas al español ${ }^{12}$.

CARRILlO, J. L. (1979), La introducción de la nueva nomenclatura y el rechazo de la teoría de la acidez de Lavoisier, Málaga, Universidad; y CARRILlO, J. L. y GAGO, R. (1980), Memoria sobre una nueva y metódica clasificación de los fluidos elásticos permanentes y gaseosos de J. M. de Aréjula, Málaga, Universidad. Un estudio detallado sobre los pensionados en Francia, con abundante y actualizada bibliografía, en GARCÍA BELMAR, A. y BERTOMEU SÁNCHEZ, J. R. (2001), «Viajes a Francia para el estudio de la química, 1770 y 1833», Asclepio, LIII-1, 95-140. Por supuesto, se hace indispensable la consulta de LÓPEZ PIÑERO, J. M ${ }^{\mathrm{a}}$.; Glick, T.; NAVARro, V.; y PORTela, E. (1983), Diccionario histórico de la ciencia moderna en España, Barcelona, Península.

10 Además de los autores ya citados, sobre este período pueden verse, para Martí i Franquès, Esqué, M. D. y CARMONA, A. M. (1995a), «La biblioteca de Martí i Franquès», en PuiGPLA, C. et alii (eds.), Actes de les III trobades d'història de la ciència i de la tècnica als Països Catalans, Barcelona, SCHCT, 397-402; y EsQuÉ, M. D. y CARMONA, A. M. (1995b), «Aportació dels investigadors de la història de la ciència als Països Catalans al coneixement de la figura d'Antoni Martí i Franquès», en Puig-PLA, C. et alii (eds.), Actes de les III trobades d'història de la ciència i de la tècnica als Països Catalans, Barcelona, SCHCT, 391-393. Y para Munárriz, GagO, R. (1982), «Introducción», en LAVOISIER, A. L., Tratado elemental de química, Madrid, Alfaguara, XIII-LXVIII. Una exposición más general en PORTELA (1999), p. 48.

11 En Portela, E. y Soler, A. (1992), «La química española en el siglo XIX», en LóPEZ PIÑERO, J. M. (ed.), La ciencia en la España del siglo XIX, Madrid, Marcial Pons, 85-107, p. 98, se puede observar la importancia de la producción bibliográfica sobre la química en los últimos decenios del siglo XVIII, sobre todo en comparación con el periodo posterior. Por su parte, en Bertomeu SÁnChez, J. R. y GARCÍA Belmar, A. (2000), «Los libros de texto de química destinados a estudiantes de medicina y cirugía en España (1788-1845), Dynamis, 20, 457-489, se estudian los manuales de química utilizados en los estudios de medicina y cirugía.

12 Algunas de las obras más importantes de este momento han sido estudiadas desde el punto de vista de la historia de la lengua por este grupo investigador. Sirvan como ejemplo GARRIGA, C. (1996), «Apuntes sobre la incorporación del léxico de la química al español, la influencia de Lavoisier», Documents pour l'histoire du français langue étrangère ou seconde, 18, 419-435; y GARRIGA, C. (1997), «La recepción de la Nueva nomenclatura química en español», Grenzgänge, 8, 33-48. Sobre la traducción de la nomenclatura química y su recepción en español, y sobre diversos textos químicos, se puede ver GARRIGA, C. (1998a), «Quí- 
Para esta tarea, el filólogo cuenta con obras lingüísticas de referencia con las que contrastar lo hallado en los textos, como son los diccionarios del siglo XVIII y comienzos del siglo XIX. Cabe destacar, en este sentido, la labor realizada por la Real Academia Española, que a partir de su primer diccionario, conocido como Diccionario de Autoridades ${ }^{13}$, asume el carácter de organismo normativo. El siglo XVIII está marcado por la intención de diferenciar el léxico común del «facultativo», de manera que la propia Academia se plantea elaborar otro diccionario que reúna «las voces propias pertenecientes a las Artes liberales y mechánicas» ${ }^{14}$. Sin embargo, lo cierto es que el diccionario contenía numerosos tecnicismos, y entre ellos, abundantes voces del campo de la química15.

Pero la Academia no llegará a cristalizar el proyecto mencionado. Será Terreros quien elabore un Diccionario castellano con las voces de ciencias y

mica, enseñanza y divulgación de la terminología, las Lecciones de química teórica y práctica de Morveau, Maret y Durande», en Brumme, J. (ed.), La historia de los lenguajes iberorrománicos de especialidad (siglos XVII-XIX); soluciones para el presente, Barcelona, UPF, 163174; los Anales de Proust en Garriga, C. (1998b), «Luis Proust y la consolidadción de la terminología química en español», en GarcíA, J. L.; Moreno, J. M.; RuIz, G. (eds.), VI Congreso de la Sociedad Española de Historia de las Ciencias y de las Técnicas, Segovia, Junta de Castilla y León, 691-699; diversos textos de Carbonell, en GuTiÉRrez CuAdRAdo, J. (1998b), «F. Carbonell y Bravo y su texto Curso analítico de química escrito en italiano por F. Mojón», en García Turza, C.; GonzÁlez, F.; MANGado, J. (eds.), Actas del IV Congreso Internacional de Historia de la Lengua Española, Logroño, AHLE - Gobierno de La Rioja Universidad de La Rioja, 219-230; y BAJO, F. (1999), «El Arte de hacer y conservar el vino de Francisco Carbonell y Bravo, primer manual de enología científica española», en BRUMME, J. (ed.), La historia de los lenguajes iberorrománicos de especialidad, la divulgación de la ciencia, Barcelona - Frankfort del Meno - Madrid, Universitat Pompeu Fabra - Vervuert Iberoamericana, 131-144; la traducción del Diccionario universal de química de Brisson en GARriga, C. (1998c), «El Diccionario Universal de Física de Brisson (1796-1802) y la fijación lexicográfica de la terminología química en español», en GARCÍA TURZA, C.; GONZÁLEZ, F.; Mangado, J. (eds.), Actas del IV Congreso Internacional de Historia de la Lengua Española, Logroño, AHLE - Gobierno de La Rioja - Universidad de La Rioja, 179-190, etc.

13 R.A.E. (1726-1739), Diccionario de la lengua castellana, Madrid, Francisco del Hierro; facsímil en Madrid, Gredos, 1984.

14 En el prólogo del primer tomo (p. V) así lo declara, y luego insiste en esta idea de nuevo en el tomo VI (p. XX). RAE (1726-1739).

15 ClÉMENT, J. P. (1993), Las instituciones científicas y la difusión de la ciencia durante la ilustración, Madrid, Akal, p. 49, señala que este primer diccionario recoge numerosos tecnicismos de la época. Para la presencia del léxico químico en Autoridades, véase el estudio de GARRIGÓs, Ll. (1990), Contribución al estudio de la constitución del lenguaje químico en castellano, Valencia, Universidad (edición en microficha). 
artes, donde se recoge el léxico «de las ciencias y artes mecánicas y liberales» que la Academia dice desatender ${ }^{16}$. Se trata, por tanto, de un repertorio útil para el estudio de este léxico, aunque hay que tener en cuenta, por un lado, que la fecha real de elaboración del diccionario fue 1745 - a pesar de que no se publicó hasta 1786-, y que el propósito de Terreros no era elaborar un diccionario «de voces de ciencias y artes», sino un diccionario «con voces de ciencias y artes» ${ }^{17}$.

Otro hecho importante en lo lingüístico sucede hacia los años en que se está proponiendo la nueva nomenclatura. Se trata de la publicación de la primera edición del Diccionario de la Real Academia en un tomo, ante la lentitud de los trabajos de revisión de Autoridades ${ }^{18}$. A partir de ahí, el Diccionario en un tomo se va reeditando, con la incorporación de las revisiones que se iban haciendo para la segunda edición de Autoridades. Así aparecen las ediciones del DRAE de 1783, 1791 y 1803, aunque habrá que esperar a la de 1817 para que se aprecien cambios sustanciales.

El cambio de siglo no constituyó en sí mismo el fin de un periodo, sino que el cultivo de las ciencias mantuvo su importancia aun cuando la crisis de la monarquía era más que evidente, como ya han puesto de manifiesto los historiadores de la ciencia ${ }^{19}$. La periodización propuesta por López Piñero sitúa en 1808 el punto de inflexión ${ }^{20}$.

16 Terreros, E. (1786), Diccionario castellano con las voces de ciencias y artes, Madrid, Vda. de Ibarra; facsímil en Madrid, Arco-Libros, 1987.

17 Dice Terreros (1786), p. XIV, en su prólogo: «Es cosa tambien muy cierta que hay multitud de máquinas, invenciones y noticias, con que la curiosidad y luces de nuestro siglo y los inmediatos nos han enriquecido, que no conocieron los pasados, de modo que apénas hai arte ni ciencia que no haya adelantado en su esfera y ganado mucho terreno, al mismo tiempo que es sin controversia que á cada invencion, á cada máquina y pieza de ellas es menester acomodarle algun nombre». Véanse también Alvar EzQuerRA, M. (1993), «El Diccionario de Terreros», Lexicografía descriptiva, Barcelona, Biblograf, 249-259, p. 254.

18 La Academia había editado el primer tomo revisado de Autoridades en 1770. Las razones de la decisión de la Academia se hallan en el «Prólogo» a la primera edición, en R.A.E. (1780), Diccionario de la lengua castellana, Madrid, J. Ibarra; facsímil en Madrid, EspasaCalpe. También resulta interesante el estudio a la edición facsímil elaborado por SECO, M. (1991), «Introducción», en R.A.E (1780).

19 En LÓPEZ PiÑERO, J. M. (1982a), «La literatura científica en la España contemporánea», en DíAZ Plaja, G. (dir.), Historia general de las literaturas hispánicas (vol. VI), Barcelona, Vergara, 675-693, p. 677, se explica la situación contradictoria que se produjo en la ciencia española, que estaba dando sus mejores frutos en las últimas décadas del siglo XVIII y primeros años del XIX, cuando el espíritu ilustrado que los había propiciado estaba en plena crisis. Las relaciones entre ciencia y universidad en este periodo han sido estudiadas en el 
Por lo que respecta a la química, mantiene el digno nivel de los últimos años del siglo XVIII, aunque como señalan Portela y Soler a partir del estudio de la producción bibliográfica, desde 1790 la publicación de obras químicas va decreciendo, para caer estrepitosamente entre 1806 y $1810^{21}$. Precisamente estos primeros años del siglo XIX son estudiados por $\mathrm{Gago}^{22}$, quien describe la evolución de los centros más importantes para el cultivo de la química con los personajes más representativos, como Proust en su peregrinaje por Vergara -donde también se ubican Chabaneau y Jerónimo Mas-, Segovia — con su alumno Munárriz - o Madrid — donde también ejercía Gutiérrez Bueno-; Aréjula y Rodríguez Jaén en Cádiz; Otano, Andreu y Brunete en Zaragoza; Ponce de León en Granada; Villanova en Valencia o Carbonell en Barcelona. Además, se mencionan las obras publicadas por estos autores y utilizadas, en muchos casos, como textos oficiales para la enseñanza, lo que proporciona pistas importantes para el filólogo a la hora de seleccionar los textos más representativos para el estudio del léxico.

Durante estos primeros años del siglo, las actitudes lingüísticas frente a la llegada de galicismos a la lengua española se mantiene en la línea de lo sucedido en el siglo XVIII. La necesidad de traducciones rápidas y poco cuidadas de todo tipo de textos, especialmente los de ciencia, hace cundir la alarma en ciertos autores, y aumenta la conciencia lingüística que en muchos casos llega a un rechazo de las aportaciones léxicas provenientes del francés ${ }^{23}$. En efecto, los textos químicos de finales del siglo XVIII y de los primeros años del siglo

clásico trabajo de Peset, M. y PeSET, J. L. (1974), La universidad española (siglos XVIIIXIX), Madrid, Taurus; y reformulada después en PESET, J. L. (1988), «Educación y ciencia en el fin del Antiguo Régimen», en SÁnchez Ron, J. M. (ed.), Ciencia y sociedad en España, de la Ilustración a la Guerra Civil, Madrid, El Arquero, 17-25, para el tránsito entre universidad antigua y liberal, y en PESET, M. y PESET, J. L. (1992), «Las universidades españolas del siglo XIX y las ciencias», en LóPEZ PIÑERO, J. L. (ed.), La ciencia en la España del siglo XIX, Madrid, Marcial Pons, 19-49, para todo el siglo XIX.

20 López Piñero propone una periodización basada en la historia de la medicina, pero que va haciendo extensiva al resto de las ciencias. Véase, a este respecto, por ejemplo, LóPEZ PIÑERO, J. M. (1982a), LÓPEZ PIÑERO, J. M. (1982b), La ciencia en la historia hispánica, Barcelona, Salvat; LÓPEZ PIÑERO, J. M. (1992), etc., donde a su vez se pueden hallar otras referencias del mismo autor.

21 Portela, E. y Soler, A. (1992) comprueban la validez de la periodización de López Piñero para el siglo XIX en relación a la química.

22 GaGO, R. (1988b), «Cultivo y enseñanza de la química en la España de principios del siglo XIX», en SÁnchez Ron, J. M. (ed.), Ciencia y sociedad en España, de la Ilustración a la Guerra Civil, Madrid, El Arquero, 129-142.

23 Véase, a este respecto, LÁZARO CARRETER, F. (1985), p. 276 y ss. 
XIX registran en sus prólogos numerosas consideraciones acerca de la lengua utilizada ${ }^{24}$.

En lo que respecta a la labor lexicográfica, vale la pena tener en cuenta el diccionario francés-español de Capmany, donde se pueden documentar numerosas voces técnicas ${ }^{25}$. También conviene destacar la traducción de algún diccionario especializado, como el Diccionario universal de física de Brisson, donde se recogen las voces más actuales de la física y de la química vertidas al español ${ }^{26}$. La Academia, por su parte, no da a la luz una nueva edición hasta varios años después de acabada la contienda. Se trata de la $5^{a}$ edición ${ }^{27}$, en la que se constata la renuncia a seguir con la reedición del diccionario de $\mathrm{Au}$ toridades, de manera que el diccionario en un tomo se convierte en la obra lexicográfica normativa de referencia para la lengua española ${ }^{28}$. Pero estas circunstancias van acompañadas de cambios más profundos en la estructura del diccionario, especialmente en lo que se refiere a los tecnicismos ${ }^{29}$. Esta

24 Véanse, por ejemplo, las consideraciones acerca de la lengua recogidas en las traducciones de la nomenclatura química al español en GARRIGA, C. (1997), p. 37 y ss.

25 Capmany, A. (1805), Nuevo diccionario francés español, Madrid, Imprenta de Sancha. El diccionario incluye un Suplemento al que se destierran las voces de ciencias. Dice Capmany (p. XV) que «el diccionario de la fisica, la chîmica, la anatomía, la medicina, la farmácia, la botánica, la pintura, y arquitectura, es de todas las naciones cultas», y sus voces se pueden adaptar a cualquier lengua con solo cambiar las terminaciones; de esta manera contradice a los que afirman que la lengua castellana es estéril para las materias filosóficas y científicas. Sobre esta obra, véase el trabajo de RoIG, C. (1995), «El Nuevo diccionario francés-español de Antonio de Capmany», en LAFARGA, F. y otros (eds.), La traducción. Metodología-historia-literatura. Ámbito hispanofrancés. Barcelona, PPU, 75-80; y referido al léxico químico, ANGLADA, E. (1997-98), «Neologismos de la química en el Nuevo diccionario francés-español de A. de Capmany», Revista de lexicografía, 4, 31-47.

26 En GARRIGA, C. (1998c) se describe la obra globalmente y se analizan algunas de las voces químicas que contiene. La nomenclatura química que el traductor añade a la obra se estudia en GARRIGA, C. (1997).

27 R.A.E. (1817), Diccionario de la lengua castellana, Madrid, Imprenta Real, $5^{\mathrm{a}}$ ed.

28 El CONDE DE LA ViÑAZA (1893), «Libro Tercero, Del Diccionario», Biblioteca histórica de la filología castellana, Madrid, Manuel Tello, 721-1018; facsímil en Madrid, Atlas, 1978; p. 1508, dice: «Tiráronse 6.000 ejemplares, que se agotaron en muy breve tiempo, pues el público esperaba ansioso esta edición primera, que se hizo después de la invasión y guerra francesa». El «Prólogo» de la propia edición de la R.A.E. (1817) también da cuenta de estas vicisitudes.

29 Por ejemplo, se advierte en el «Prólogo» de la R.A.E. (1817) que: «Se han aclarado y rectificado muchas definiciones, especialmente en los articulos que pertenecen á ciencias naturales, en las que los adelantamientos de estos últimos tiempos han aclarado y corregido diferentes equivocaciones que antes eran comunes». La Corporación justifica el aumento de 
revisión tiene una incidencia especial en algunos conceptos nuevos de la nomenclatura química que aún no se habían recogido en el diccionario, y en otros términos tradicionales como aire y agua que cambian su significado a raíz de los nuevos hallazgos químicos ${ }^{30}$.

Esta edición se encabalga con el llamado «periodo catástrofe» de la ciencia española contemporánea, que se inicia con la Guerra de la Independencia y el reinado de Fernando VII. La química también refleja ese colapso que se observa en otras ciencias, como muestran los recuentos bibliográficos de Portela y Soler ${ }^{31}$. En este periodo solo destaca un químico, Orfila, pero sus numerosos textos son de poco interés para la historia de la lengua española, ya que su obra estaba fundamentalmente en francés.

Tras el reinado de Fernando VII, con la vuelta de los exiliados liberales, la situación de la ciencia mejora apreciablemente: aumentan las publicaciones y las traducciones, se empieza a desarrollar el periodismo científico, circulan los libros extranjeros, se crean instituciones científicas... Se abre lo que López Piñero llama la «etapa intermedia» (1833-1875)32. También en este periodo se acomete, aunque con titubeos, la reforma de las enseñanzas universitarias, dando más protagonismo a las ciencias ${ }^{33}$. La química aparece junto a la física en las primeras cátedras, desempeñadas por personajes como González Valledor, Santiago Masarnau o Andrés Alcón. Pero la situación de estas prime-

voces técnicas porque «(...) la autoridad de nuestros mejores escritores ó el uso comun, constante y continuado de las personas cultas obliga á admitir(las) en el Diccionario, á pesar de la pausada circunspeccion con que en esto procede la Academia, y que quizá parecerá á algunos excesiva». Para el debate que a partir de esta edición se inicia sobre la conveniencia o no de abrir el Diccionario a los tecnicismos y darle un carácter más enciclopédico, véase AlvAR, M. (1992), «El caminar del diccionario académico», en Euralex'90, Proceedings, Barcelona, Biblograf, 327; AngladA, E. y BARgalló, M. (1992), «Principios de lexicografía moderna en diccionarios del siglo XIX», II Congreso Internacional de Historia de la Lengua Española, Madrid, Pabellón de España, 955-962; y los ya clásicos trabajos de SECO, M. (1987a), «El nacimiento de la lexicografía moderna no académica», en Estudios de lexicografia española, Madrid, Paraninfo, 129151; y SECO, M. (1987b), «Ramón Joaquín Domínguez», en Estudios de lexicografía española, Madrid, Paraninfo, 152-164. En BATTANER, M. P. (1996), «Terminología y diccionarios», en Jornada Panllatina de Terminologia, Barcelona, Universitat Pompeu Fabra, 93-117, se estudia la evolución de las voces científicas y técnicas en el Diccionario de la Academia.

30 Acerca de las voces químicas en la edición de 1817, véase GARRIGA, C. (1996-97), «Penetración del léxico químico en el DRAE, la edición de 1817», Revista de Lexicografia, 3, 59-80.

31 Portela, E. y SOlER, A. (1992).

32 LóPEZ PIÑERO, J. M. (1982a), p. 679. De nuevo resultan elocuentes los índices sobre producción bibliográfica ofrecidos por PORTELA, E. y SOLER, A. (1992).

33 Véase Peset, M. y Peset, J. L. (1992), p. 25. 
ras cátedras era muy precaria, y no incorporaban las innovaciones científicas propias del momento, sino que tenían una finalidad divulgadora de conocimientos útiles para alumnos que no se iban a dedicar a la química ${ }^{34}$.

En los años siguientes, la situación va mejorando, debido básicamente a la creciente presencia de la química en la universidad. Los nombres de Antonio Casares, Mariano Echevarría, Torres Muñoz de Luna, Sáenz Díez, Sáez Palacios, Montells y Nadal, Bonet y Bonfill, etc., están unidos a un esfuerzo de normalización de la enseñanza de la química. Se traducen las principales obras de Berzelius, Liebig, Dumas, Fresenius.... Todos ellos son textos de gran utilidad para la descripción del desarrollo lingüístico de la química a mediados de $\operatorname{siglo}^{35}$. Es importante señalar, en este sentido, un fenómeno también relevante para la historia de la lengua técnica: las dos terceras partes de los textos químicos traducidos en este periodo son franceses, y solo un diez por ciento se vierte desde el alemán. Este hecho contrasta con la situación real de la investigación de la época, en la que el predominio alemán era abrumador $^{36}$. La consecuencia fue que el español siguió bajo la influencia del

34 Moya, T. (1994), «La enseñanza de la química en la universidad española del siglo XIX», en Asclepio, XLVI-2, 43-57; p. 45.

35 Análisis de datos bibliográficos de nuevo en Portela, E. y Soler, A. (1992), p. 102 y ss. Aspectos relacionados con las decisiones terminológicas de Torres Muñoz de Luna, en GutiéRREZ CuADRADO, J. (1998a), «Torres Muñoz de Luna y la lengua de la química en el siglo XIX», en García, J. L.; Moreno, J. M.; RuIZ, G. (eds.), VI Congreso de la Sociedad Española de Historia de las Ciencias y de las Técnicas, Segovia, Junta de Castilla y León, 701-711. La traducción del Tratado de química orgánica de Liebig está estudiada en GARRIGA, C. (2001b), «Notas sobre el vocabulario de la química orgánica en español, Liebig y la divulgación de los derivados en -ina», en BARgalló, M.; ForgAS, E.; GARRIGA, C.; SCHNITZER, J.; RUBIO, A. (eds.), Las lenguas de especialidad y su didáctica, Tarragona, Universitat Rovira i Virgili, 169-180; y la del Léxico histórico y sinonímico de Hoefer en GARRIGA, C. (2002a), «Notas sobre la incorporación de los términos de elementos químicos en español en el s. XIX, el Léxico histórico y sinonímico de Ferdinand Hoefer», en PöLl, B. y RAINER, F. (eds.), Vocabula et vocabularia. Études de lexicologie et de (méta-)lexicographie romanes en l'honneur du 60e anniversaire de Dieter Messner, Frankfort del Meno, Peter Lang, 131-144. A estos textos cabría añadir las traducciones de Daguerre, estudiadas por GÁlLEGO, R. (2002), «Notas sobre la historia del léxico de la fotografía en español», en ECHENIQUE, M. T. y SÁNCHEZ, J. (eds.), Actas del V Congreso internacional de historia de la lengua española, Madrid, Gredos, 2051-2062, que aunque tenían finalidades técnicas aplicadas a la fotografía, también son de gran interés para la historia de la lengua.

36 El dato está extraído de Portela, E. y Soler, A. (1992), p. 100. Estos autores explican la situación debido a la dependencia de la química española respecto de la francesa en el momento de la desconexión, lo que habría llevado a retormar los mismos canales de influencia en el momento de la reincorporación. En efecto, incluso un manual original alemán como 
francés más allá de lo esperable, en lo que a lenguaje científico y técnico relacionado con la química se refiere.

Otro aspecto de relevancia para la historia de la lengua española es la aparición, a mediados de siglo, de una importante actividad lexicográfica de tendencia enciclopedista, paralela a la académica, que busca aumentar el caudal del repertorio léxico oficial a través de la incorporación de voces técnicas. Los diccionarios de autores como Salvá, Domínguez, Chao, etc., permiten documentar las voces de ciencias y técnicas mucho antes de que las acoja el diccionario académico ${ }^{37}$. En este sentido, el léxico químico adquiere un protagonismo que no tenía hasta ese momento, consolidando así su presencia en la lengua.

El último cuarto del siglo XIX, periodo denominado «la generación de los sabios», se caracteriza por la extensión en el estudio y la práctica de la ciencia y por la aparición de investigación original, ausente desde la Ilustración ${ }^{38}$. Sin embargo, en España la química no se desarrolla al mismo ritmo que otras ciencias, dada la precariedad de los laboratorios universitarios y la complejidad a la que la ciencia había llegado, así como la necesidad de formar equipos profesionalizados que fueran más allá del esfuerzo aislado de un científico. Los químicos más sobresalientes de este periodo, como Casares Gil, Rodríguez Carracido o Gregorio Rocasolano proyectan sus trabajos ya en el siglo $\mathrm{XX}$, por lo que quedan fuera del ámbito de este estudio.

el Tratado de química orgánica de Liebig se traduce al español desde la traducción francesa de Gerhardt, como se muestra en GARRIGA, C. (2001b), p. 170. Esta situación lleva a GuTIÉRREZ CUADRADO, J. (2001), p. 184, a describir la evolución de la lengua química en español como la historia de una dependencia.

37 Estos aspectos están tratados en SECO, M. (1987a), BAQUERO R. (1992), «Notas en contribución a la historia de la lexicografía española monolingüe del siglo XIX», en Euralex'90, Proceedings, Barcelona, Biblograf, 455-461; AngladA, E. y BArgalló, M. (1992), o Azorín, D. (1996), «La lexicografía española en el siglo XIX, desarrollos y tendencias», en SERRA ALEGRE, E. et al. (eds.), Panorama de la investigació lingüística a l'Estat Espanyol, València, Universitat de València, 48-54. Algunos estudios de estos autores en SECO, M. (1987b) para R. J. Domínguez; para Salvá, AzORÍN, D. y BAQUERO, R. (1994-95), «De la teoría a la práctica lexicográfica, el «Nuevo Diccionario de la Lengua Castellana» de Vicente Salvá», Estudios Lingüisticos de la Universidad de Alicante, 10, 9-20; para el editado por Gaspar y Roig, BUENO, A. Mª (1996), «La lexicografía no académica del siglo XIX, el Diccionario Enciclopédico de la Lengua Española publicado por la editorial Gaspar y Roig», en Alvar EzQUeRRA, M. (coord.), Estudios de Historia de la Lexicografia del Español, Málaga, Universidad, 151-157, etc. Para el léxico químico, resulta especialmente interesante el de IGLESIA, S. (2002), Las voces de la química en el Diccionario Nacional de R. J. Domínguez, la marcación y la definición, Barcelona, Universitat Pompeu Fabra (trabajo de investigación).

38 LÓPEZ PIÑERO, J. M. (1982a), p. 684. 
Lingüísticamente, cabe mencionar la transformación que el diccionario académico experimenta a partir, sobre todo, de la duodécima edición ${ }^{39}$ con la incorporación de voces técnicas en su repertorio, y que se proyecta en la última edición del siglo ${ }^{40}$. Paralelamente se siguen produciendo diccionarios enciclopédicos en los que el léxico científico y técnico se recoge en abundancia ${ }^{41}$, y se debate abiertamente sobre temas relacionados con el lenguaje científico y la adaptación de tecnicismos, con el telón de fondo de la unidad del idioma ${ }^{42}$.

\section{HiSTORIA DE LA LENGUA}

La renovación léxica en una lengua se manifiesta de manera más clara en la adopción de nuevos términos que hasta ese momento no existían, pero no es menos importante el cambio de signficado que se produce en algunas voces tradicionales debido a los avances científicos. Por último, los procesos de formación de palabras también son aprovechados por la lengua para la creación de nuevos términos. A continuación mostraré algunos ejemplos de estos tres fenómenos.

39 R.A.E. (1884), Diccionario de la lengua castellana, Madrid, Gregorio Hernando, $12^{\mathrm{a}}$ ed.

40 R.A.E. (1899), Diccionario de la lengua castellana, Madrid, Hernendo y compañía, $13^{\mathrm{a}}$ ed. En GARRIGA, C. (2001a), «Sobre el diccionario académico, la 12a ed. (1884)», en MEDINA Guerra, A. M. (ed.), Estudios de lexicografía diacrónica del español, Málaga, Universidad, 261-315, se describe la edición de 1884 y se pone de manifiesto el aumento de voces técnicas en el vocabulario académico. Para la edición de 1899, véase CLAVERÍA, G. (2001), «El léxico especializado en la lexicografía de finales del siglo XIX, la decimotercera edición (1899) del Diccionario de la Lengua Castellana de la Real Academia», en BRUMME, J. (ed.), La historia de los lenguajes iberorrománicos de especialidad, la divulgación de la ciencia, Barcelona - Frankfort del Meno - Madrid, Universitat Pompeu Fabra - Vervuert - Iberoamericana, 207-222.

41 Destaca entre ellos el Diccionario hispanoamericano, estudiado por GUTIÉRREZ CUADRADO, J. (1994), «El Diccionario Hispano-americano de Montaner y Simón», Actas del XXIX Congreso del Instituto Internacional de Literatura Iberoamericana, Barcelona, Universidad, 263-282.

42 Véanse, por ejemplo, las intervenciones de personajes como José Carvajal, Lasso de la Vega, Jacinto Bonilla, Ramón Arizcún, Román Oriol o del mismo Rodríguez Carracido en el Congreso Literario Hispano-ámericano de 1892 (ASOCIACIÓN DE ESCRITORES Y ARTISTAS ESPAÑOLES (1893), Congreso Literario Hispano-americano, Madrid, Ricardo Fé [edición facsímil en Madrid, Instituto Cervantes - Pabellón de España - Biblioteca Nacional, 1992]). A este respecto, véase GutiérRez CUADRADO, J. y PASCUAL, J. A. (1992), «Prólogo a propósito de las Actas del Congreso Literario Hispano-americano de 1892», en ASOCIACIÓN DE ESCRITORES Y ARTISTAS ESPAÑOLES (1893), IX-XXXI. 


\subsection{Nuevos términos}

La aparición de un nuevo término produce tensiones en la lengua encaminadas a la reorganización del sistema. Además, hasta que ese nuevo término se asienta en el uso, frecuentemente debe competir con otras propuestas léxicas que aspiran a ocupar el mismo espacio lingüístico. Aquí se trata un ejemplo muy característico que, aunque se acuña en el último cuarto del siglo XVIII, se proyecta como ningún otro en la química del siglo XIX: el de oxígeno ${ }^{43}$.

Este término es seguramente el más conocido de los introducidos por Lavoisier y sus colaboradores, aunque el descubrimiento se debió a Priestley, químico partidario de la teoría del flogisto, quien le dio el nombre de aire desflogisticado. El Diccionario de la Academia lo recoge en la $5^{\text {a }}$ edición ${ }^{44}$, pero para entonces el término estaba sobradamente consolidado, tras haber competido con otros términos rivales. Además de la denominación de Priestley, recibía los nombres de aire puro y aire vital, y debido a su importancia en la teoría de la acidez de Lavoisier, este autor lo llamó también principio acidificante. La primera documentación hallada de la voz oxígeno en español se encuentra en un texto de Martí i Franquès (1787), quien afirma que:

El acido fosforico, el vinagre, i finalmente todos los acidos encierran gran cantidad de aire puro, por cuia razon lo llama el Sor Lavoisier principio oxygino, o acidificante ${ }^{45}$.

Y solo un año después ya aparece en la traducción del Método de la nueva nomenclatura química que traduce Gutiérrez Bueno ${ }^{46}$ así como en las Reflexiones de Aréjula ${ }^{47}$, aunque fuera para rechazarlo. La razón estaba en la constatación de que el oxígeno no era un 'generador de ácidos', como Lavoi-

43 Se pueden hallar los ejemplos hidrógeno y nitrógeno en GARRIGA, C. (2003), «Aspectos de la adaptación de la nueva nomenclatura química al español», GUTIÉRREZ, B. (ed.), Aproximaciones al lenguaje de la ciencia, Salamanca, Universidad, en prensa.

44 R.A.E. (1817).

45 MARTí I FrANQUÈS, A. (1787), «Sobre algunas producciones que resultan de la combinación de varias sustancias aeriformes», en QuINTANA, A. (1935), Antoni de Martí i Franquès; memòries originals, estudi biogràfic $i$ documental, Barcelona, Acadèmia de Ciències i Arts, 11-20, p. 19.

46 Morveau, L.; Lavoisier, A. L.; Berthollet, C. y Fourcroy, F. (1788), Método de la nueva nomenclatura química, Madrid, Antonio de Sancha.

47 ARÉJUla, J. M. (1788), Reflexiones sobre la nueva nomenclatura química, Madrid, A. de Sancha; facsímil en R. Gago y J. L. Carrillo (1979), La introducción de la nueva nomenclatura y el rechazo de la teoría de la acidez de Lavoisier en España, Málaga, Universidad, p. 2. 
sier decía al proponer el término. Así, además de las lexías formadas a partir de aire (desflogisticado, puro y vital) y de la forma principio acidificante, el término oxígeno compite en español con las siguientes propuestas léxicas:

- arxicayo. La propuesta es de Aréjula (1788), quien obtenía la palabra de acuerdo la etimología griega ( $\alpha \rho \kappa \eta$ 'principio' у к $\alpha \iota \alpha \nu$ 'quemante') ${ }^{48}$. El Diccionario de la Academia no llegará a incluir este término, aunque sí que se encuentra en el Diccionario enciclopédico de E. Chao, definido como 'Quím. ant.: gas oxíjeno'49.

- gas comburente. Lo propone Porcel ${ }^{50}$, de acuerdo con las observaciones de Aréjula, argumentando como ventaja frente a arxicayo que la voz comburente ya existía en español, lo que hacía innecesario crear otra nueva ${ }^{51}$. El Diccionario de la Academia no registra comburente, pero se puede hallar en el Diccionario Nacional de Domínguez ${ }^{52}$ y en el Enciclopédico de Chao53: 'Quím.: lo que combinándose con otro cuerpo, produce el fenómeno de la combustión; calificación que merece solo el oxíjeno (...)'.

- gas pyrógeno. Esta solución, que busca la analogía con otros términos como oxígeno e hidrógeno, es propuesta por Chabaneau, también de acuerdo con las objeciones de Aréjula ${ }^{54}$. Pero la voz pyrogeno no se ha llegado a documentar en ningún diccionario del siglo XIX.

A estos autores, sin embargo, se les escapaba que lo fundamental de un término científico no es que su significado responda a la etimología de sus formantes, sino que la comunidad científica esté de acuerdo en atribuirle ese valor. $\mathrm{Y}$ eso ocurrió con oxígeno, que debido a su rápida divulgación, se había asentado ya entre la comunidad científica, y no se vería desbancado por ninguno de sus competidores.

48 ArÉJUla, J. M. (1788), p. 23.

49 ChaO, E. (1864), Diccionario Enciclopédico de la Lengua Española, Madrid, Gaspar y Roig.

50 PorCel, T. A. (1788), p. 42.

51 Acerca de este episodio, desde la perspectiva filológica, véase GÓMEZ DE ENTERRÍA, J. (1998), «Consideraciones sobre la terminología científico-técnica de carácter patrimonial en el español del siglo XVIII», Boletín de la Real Academia Española, 78, 275-301, p. 296.

52 Domínguez, R. J. (1846), Diccionario nacional, Madrid, Miguel Guijarro.

53 CHAO, E. (1864).

54 Véase Gago, R. (1982), «Introducción», en LavoisieR, A. L., Tratado elemental de química, Madrid, Alfaguara, XIII-LXVIII, p. IL; y GÓMEZ DE ENTERRÍA, J. (1998), p. 297. 
Y una prueba de su consolidación en el uso es la rápida creación de derivados que aparecen desde los primeros textos ${ }^{55}$, y que paulatinamente se irán incorporando a los diccionarios del español: oxigenación, oxigenado, oxigenar, desoxigenar, óxido, oxidación, oxidar, sobreoxigenación, etc. ${ }^{56}$.

\subsection{Cambio de significado en términos tradicionales}

Menos perceptible resulta siempre el cambio de significado de determinadas palabras en virtud de los progresos de la ciencia. Un caso paradigmático es el de la voz agua, considerada un elemento simple según la teoría aristotélica. Así la definía el primero de los diccionarios académicos, el de Autoridades:

Elemento principál entre los quatro. Los mas de los Philósophos sintieron era uno de los principios de la naturaleza. Dividese en dos especies, naturál, y artificiál: la naturál es la que cae de las nubes, la del mar, la de los rios, arroyos, fuentes y pozos; y la artificiál la que es compuesta, como agua de azár, agua rosada, \&c. ${ }^{57}$

Esta era también la concepción del diccionario de Terreros ${ }^{58}$. El cambio se percibe claramente en los textos de Lavoisier ${ }^{59}$, pero en español se documenta esta nueva concepción de nuevo de Martí i Franquès (1787), quien decía: «El agua pues debe ser excluida de la clase de los elementos, siendo indubitable formacion por la mescla de los aires inflamable, i desflogisticado, cuias dos substancias serán sus dos principios constitutivos, como lo jusga el Sor. Lavoisier $(\ldots))^{60}$.

55 Por ejemplo, en Proust, L. (1791), Anales del Real Laboratorio de Química de Segovia, Segovia, A. Espinosa; ed. facsímil de R. Gago, Segovia, Academia de Artillería, 1990. Véase GARRIGA, C. (1998b), p. 693.

56 Algunos de estos términos se documentan lexicográficamente en GARRIGA, C. (1996-97). También se puede hallar la forma gas arxicayado en Aréjula (1790); véase CARRILLO, J. L. y GAGO, R. (1980), p. 8. Para la presencia de los términos de elementos químicos en el diccionario académico, véase GARRIGA, C. (2002a).

57 R.A.E. (1726).

58 TERREROS, E. (1786).

59 Lavoisier expresaba que: «El agua se habia tenido hasta nuestros dias por una sustancia simple; y no halláron dificultad alguna los antiguos para calificarla con el nombre de elemento; en efecto debian tenerla por tal, pues no habian llegado a descomponerla (...)». En LAVOISIER, A. L. (1798), Tratado elemental de química [trad. por J. M. Munárriz), Madrid, Imprenta Real, p. 60.

60 MARTí I FranQUÈs, A. (1787), p. 14. 
Es de nuevo en el siglo XIX cuando los cambios se reflejan en el Diccionario. En la $4^{\mathrm{a}}$ edición agua se define como 'Cuerpo fluido, húmedo, y transparente, sin olor ni color, y capaz de apagar el fuego'61, sin hacer referencia al concepto de sustancia simple, y ya en la $5^{\text {a }}$ edición, la definición se transforma definitivamente:

Sustancia en su estado mas comun fluida, elastica, trasparente, insípida y sin olor, la mas abundante derramada por todos los cuerpos de la naturaleza. Hasta nuestros dias fue reputada por simple, y como tal por uno de los elementos ó principio de los cuerpos ${ }^{62}$.

Habrá que esperar, sin embargo, hasta la $11^{\mathrm{a}}$ edición para que el Diccionario defina agua como un compuesto de oxígeno y de hidrógeno ${ }^{63}$. De nuevo, los textos sirven para avanzar lo que se documenta en las obras lexicográficas ${ }^{64}$.

\subsection{Nuevos formantes.}

A mediados del siglo XIX se produce el momento del desarrollo de la química orgánica. Uno de los síntomas de progreso en la nomenclatura de la química orgánica fue la utilización de nombres formados con sufijos comunes para las sustancias de una misma característica, sistema inspirado, en principio, en la nomenclatura propuesta para la química inorgánica, pero desvirtuado tempranamente por su falta de sistematicidad ${ }^{65}$.

El ejemplo más claro es el del sufijo -ina, utilizado para los alcaloides desde que la traducción francesa de morphium consagró morphine (1817), y a partir de aquí se utilizara ese formante -ine como identificador de los alcaloi-

\footnotetext{
61 R.A.E. (1803), Diccionario de la lengua castellana, Madrid, Vda. de Ibarra, $4^{\mathrm{a}}$ ed.

62 R.A.E. (1817).

63 R.A.E. (1869), Diccionario de la lengua castellana, Madrid, M. Rivadeneyra, $11^{\mathrm{a}} \mathrm{ed}$.

64 Voces como aire, fuego, éter se estudian desde esta perspectiva en GARRIGA, C. (1996-97).

65 Crosland, M. (1962), p. 269. Así, en la obra de Hoefer, F. (1845), Nomenclature et classifications chimiques (...), Paris, Baillière, traducidas al español ocho años más tarde (HoEFER, F. (1853), Nomenclatura y clasificaciones químicas (...), Madrid, M. A. Gil), el autor se queja repetidamente de este hecho. Valgan como ejemplos las siguientes palabras: «Si los sucesores de Lavoisier hubieran seguido las huellas de su maestro, no estaria hoy la química orgánica entregada a la anarquía»; en HoEfER, F. (1853), p. 41. Sobre la traducción de la Nomenclature al español, véase GARRIGA (2002a).
} 
des ${ }^{66}$. La terminación -ine se traduce al español como -ina. En la adaptación al español del Tratado de química orgánica de Liebig (1847-48) ${ }^{67}$, en un capítulo del tomo III titulado «Alcaloides», se recoge y explica un total de 73 sustancias. Muchas de ellas tienen denominaciones que han quedado anticuadas, o que no han penetrado, por su especificidad, en los diccionarios generales. En cambio, otras han traspasado la frontera de la lengua científica y se usan habitualmente en la lengua común debido a sus frecuentes aplicaciones médicas.

Una de las sustancias que se documenta en Liebig es, desde luego, morfi$n a^{68}$. Sin embargo, no tendrá cabida en el Diccionario hasta la $11^{\mathrm{a}}$ ed. ${ }^{69}$. Pero no es esta la primera documentación lexicográfica del término, ya que Domínguez (1846-47) lo recoge en su diccionario. Hay que tener en cuenta que este alcaloide se descubre en 1817 , un momento en que la ciencia española estaba sumida en una grave crisis. No es de extrañar que, dada la ausencia de textos sobre la materia, la primera documentación estuviera en un diccionario enciclopédico, que, por otro lado, confiesa sus fuentes francesas ${ }^{70}$. La trayectoria académica de morfina es también característica de otros términos científicos y técnicos, que entran en las ediciones de la segunda mitad del siglo XIX, y a partir de ahí se mantienen prácticamente inalterados. Véanse las definciones:

'f. Alcali vegetal amargo que se extrae del opio'71.

'(De Morfeo, dios del sueño, á causa de la virtud soporífica de esta substancia.) f. Álcali vegetal amargo que se extrae del opio. Combinada con los ácidos, produce sales muy venenosas' 72 .

'(De Morfeo, dios del sueño, á causa de la virtud soporífica de esta substancia.) f. Alcaloide sólido, muy amargo y venenoso, que cristaliza en prismas rectos e in-

66 La sucesión de alcaloides descubiertos y cómo se llegó a sus denominaciones, en Crosland, M. (1962), p. 300.

67 LIEBIG, J. (1847-48), Tratado de química orgánica, Madrid, La Ilustración. El texto está traducido por Sáez Palacios y Ferrari Scardini. Un estudio sobre el mismo en GARRIGA, C. (2001b).

68 LieBIG, J. (1847-48), vol. III, p. 319.

69 R.A.E. (1869).

70 El diccionario de Domínguez es un caso probable de precocidad frente a los textos en la incorporación del léxico científico y técnico, ya que, como explica SECO (1987b), p. 160, Domínguez toma como fuente fundamental el diccionario de Bescherelle, editado en Francia tres años antes, y de carácter enciclopédico (téngase en cuenta el protagonismo de la química francesa en la época).

71 R.A.E. (1869).

72 R.A.E. (1884). 
coloros y transparentes; se extrae del opio, y en dosis pequeñas, se emplea en medicina como soporífero y anestésico'73.

Si se compara la definición de 1899 con la de la última edición del Diccionario, se verá que es prácticamente la misma ${ }^{74}$.

Sin embargo, la primera denominación de un alcaloide que entra en el Diccionario no es la voz morfina, sino el término quinina, introducido en la $8^{a}$ edición $^{75}$. Pero es un caso esporádico, porque no aparece ningún otro hasta la $11^{\mathrm{a}}$ edición $^{76}$, cuando junto a morfina se incorpora nicotina, y hay que esperar a la $12^{\mathrm{a}}$ edición ${ }^{77}$ para encontrar las voces atropina, codeína, estricnina, narcotina y teína, y a la $13^{\mathrm{a}}$ edición $^{78}$ para hallar cafeína y anilina. Todas estas voces están documentadas en Liebig ${ }^{79}$ y aparecen en los diccionarios de Domínguez y Chao, anticipándose siempre al repertorio académico ${ }^{80}$. Este sufijo, ya en el siglo XX, ha alcanzado cierta productividad en la lengua común, asociado al significado de 'droga' (cocaína, heroína, morfina, etc.) y de 'medicamento' (aspirina, penicilina, insulina, etc.) ${ }^{81}$.

Otro ejemplo con menos fortuna lexicográfica en el siglo XIX fue el de las sustancias con denominaciones acabadas en -ona. En este caso fue acetona el término que dio lugar a la regularización. Aunque descubierto en $1833^{82}$, y documentado en la traducción de Liebig ${ }^{83}$ y en el Diccionario de $\mathrm{Chao}^{84}$, no se encuentra en el repertorio académico hasta la $15^{\mathrm{a}}$ edición $^{85}$.

73 R.A.E. (1899).

74 R.A.E. (2001), Diccionario de la lengua española, Madrid, Espasa-Calpe, 22 ${ }^{\mathrm{a}}$ ed. Las únicas variaciones están en la etimología, y en la incorporación de la palabra medicamento antepuesta a soporífero, a partir de la edición de la R.A.E. (1925), Diccionario de la lengua española, Madrid, Espasa-Calpe, $15^{\mathrm{a}}$ ed.

75 R.A.E. (1837), Diccionario de la lengua castellana, Madrid, Imprenta Nacional, $8^{\text {a }}$ ed.

76 R.A.E. (1869).

77 R.A.E. (1884).

78 R.A.E. (1899).

79 LIEBIG, J. (1847-48).

80 DomíngueZ, R. J. (1846) y CHAO, E. (1864). También se anticipa al único diccionario con dataciones de que se dispone en español, y que debe hacer las veces de diccionario histórico: COROMINAS, J. y PASCUAL, J. A. (1980-1991), Diccionario crítico etimológico castellano e hispánico, Madrid, Gredos. Datos pormenorizados en GARRIGA, C. (2001b).

81 RAINER, F. (1993), Spanische Wortbildungslehre, Tübingen, Niemeyer, p. 545.

82 Crosland, M. (1962), p. 300.

83 LIEBIG, J. (1847-48), p. 186.

84 CHAO, E. (1853-55).

85 R.A.E. (1925). Véase un estudio de los términos en -ona en GARRIGA, C. (2002b), «Notas sobre la incorporación del sufijo técnico -ona al español», en ECHENIQUE, M. T. et al. 


\section{CONCLUSIÓN}

La historia de la lengua del siglo XIX requiere el estudio de otros textos, además de los literarios. El filólogo no puede limitarse a las obras literarias e ignorar otro ámbito de gran dinamismo para la lengua, como es la actividad científica y técnica, especialmente en un siglo en el que el avance de la ciencia y el desarrollo de la técnica se convierten en motor del conocimiento.

Es verdad que hay dificultades para acercarse a los textos técnicos y seleccionar aquellos que sean los más repesentativos para estudiarlos filológicamente, pero es indispensable, ya que no sirve quedarse en los diccionarios de lengua general que, como no puede ser de otra manera, actúan de notarios del uso y recogen las voces siempre con una acusada posterioridad respecto al momento de entrada en la lengua. Hay que acercarse a los textos. Y aquí es cuando el conocimiento de la historia de la ciencia acude en apoyo del filólogo.

Hemos visto que la lengua se renueva al ritmo que evoluciona la ciencia. La lengua no es más (ni menos) que el vehículo que sirve para expresar las nuevas ideas, los conceptos innovadores, los descubrimientos. Las traducciones de los textos orginales se convierten en vía de penetración de neologismos, dan fe de las tensiones que experimenta la lengua al dar cabida a una nueva unidad, o a varias que compiten para acabar imponiéndose una de ellas. Pero a veces este mecanismo es más sutil, y consiste en cambiar el concepto de una palabra que ya existe desde hace largo tiempo en la lengua. Otras veces, el fenómeno consiste en penetrar hasta los más esenciales mecanismos léxicos e introducir nuevos elementos en el sistema que, mediante los procesos de formación de palabras, den lugar a nuevos términos. Aquí se han repasado algunos ejemplos de estos fenómenos, que muestran cómo se renovó el léxico científico del español del siglo XIX.

\section{BIBLIOGRAFÍA}

Alvar M. (1992), «El caminar del diccionario académico», en Euralex’90, Proceedings, Barcelona, Biblograf, pp. 3-27.

Alvar EzQuerra, M. (1993), «El Diccionario de Terreros», Lexicografía descriptiva, Barcelona, Biblograf, pp. 249-259.

(eds.), Actas del V Congreso Internacional de Historia de la Lengua Española, Madrid, Gredos, 2093-2106. 
ANGLADA, E. (1997-98), «Neologismos de la química en el Nuevo diccionario francésespañol de A. de Capmany», Revista de lexicografía, 4, pp. 31-47.

AngladA E. y BARGALló, M. (1992), «Principios de lexicografía moderna en diccionarios del siglo XIX», II Congreso Internacional de Historia de la Lengua Española, Madrid, Pabellón de España, pp. 955-962.

ARÉJULA, J. M. (1788), Reflexiones sobre la nueva nomenclatura química, Madrid, A. de Sancha; facsímil en R. Gago y J. L. Carrillo (1979), La introducción de la nueva nomenclatura y el rechazo de la teoría de la acidez de Lavoisier en España, Málaga, Universidad.

ASOCIACIÓN DE ESCRITORES Y ARTISTAS ESPAÑOLES (1893), Congreso Literario Hispano-americano, Madrid, Ricardo Fé (edición facsímil en Madrid, Instituto Cervantes Pabellón de España - Biblioteca Nacional, 1992).

AZORín D. (1996), «La lexicografía española en el siglo XIX, desarrollos y tendencias», en Serra Alegre, E. et al. (eds.), Panorama de la investigació lingüística a l'Estat Espanyol, València, Universitat de València, pp. 48-54.

AZORÍN D. y BAQUERO, R. (1994-95), «De la teoría a la práctica lexicográfica, el «Nuevo Diccionario de la Lengua Castellana» de Vicente Salvá», Estudios Lingüísticos de la Universidad de Alicante, 10, pp. 9-20.

BAJO, F. (1999), «El Arte de hacer y conservar el vino de Francisco Carbonell y Bravo, primer manual de enología científica española», en BRUMME, J. (ed.), La historia de los lenguajes iberorrománicos de especialidad, la divulgación de la ciencia, Barcelona - Frankfort del Meno - Madrid, Universitat Pompeu Fabra - Vervuert - Iberoamericana, pp. 131-144.

BAQUERO R. (1992), «Notas en contribución a la historia de la lexicografía española monolingüe del siglo XIX», en Euralex'90, Proceedings, Barcelona, Biblograf, pp. 455-461.

BATTANeR, M. P. (1996), «Terminología y diccionarios», en Jornada Panllatina de Terminologia, Barcelona, Universitat Pompeu Fabra, pp. 93-117.

BERTOMEU SÁNCHEZ, J. R. (1994), «Los cultivadores de la ciencia españoles y el gobierno de José I (1808-1813). Un estudio prosopográfico», Asclepio, XLVI-1, pp. 125-155.

Bertomeu SÁnchez, J. R. y García Belmar, A. (1995), «Alumnos españoles en los cursos de química del Collège de France (1774-1833)», en PUIG-PLA, C. et alii (eds.), Actes de les III trobades d'història de la ciència i de la tècnica als Països Catalans, Barcelona, SCHCT, pp. 407-418.

Bertomeu SÁnchez, J. R. y GARcía Belmar, A. (2000), «Los libros de texto de química destinados a estudiantes de medicina y cirugía en España (1788-1845), Dynamis, 20, pp. 457-489.

BuEnO A. Mª (1996), «La lexicografía no académica del siglo XIX, el Diccionario Enciclopédico de la Lengua Española publicado por la editorial Gaspar y Roig», en ALVAR EZQUERRA, M. (coord.), Estudios de Historia de la Lexicografía del Español, Málaga, Universidad, pp. 151-157.

CAPMANY, A. (1805), Nuevo diccionario francés español, Madrid, Imprenta de Sancha. 
CARrillo, J. L. y GAgo, R. (1980), Memoria sobre una nueva y metódica clasificación de los fluidos elásticos permanentes y gaseosos de J. M. de Aréjula, Málaga, Universidad.

CHAO, E. (1864), Diccionario Enciclopédico de la Lengua Española, Madrid, Gaspar y Roig.

ClaveríA, G. (2001), «El léxico especializado en la lexicografía de finales del siglo XIX, la decimotercera edición (1899) del Diccionario de la Lengua Castellana de la Real Academia», en BRUMME, J. (ed.), La historia de los lenguajes iberorrománicos de especialidad, la divulgación de la ciencia, Barcelona - Frankfort del Meno - Madrid, Universitat Pompeu Fabra - Vervuert - Iberoamericana, pp. 207-222.

ClÉMENT, J. P. (1993), Las instituciones científicas y la difusión de la ciencia durante la ilustración, Madrid, Akal.

CONDE DE LA VIÑAZA (1893), «Libro Tercero, Del Diccionario», Biblioteca histórica de la filología castellana, Madrid, Manuel Tello, pp. 721-1018; facsímil en Madrid, Atlas, 1978.

Corominas, J. y PASCUAL, J. A. (1980-1991), Diccionario crítico etimológico castellano e hispánico, Madrid, Gredos.

Crosland, M. P. (1962), Historical Studies in the Language of Chemistry, Londres, Heinemann Educational Books.

DomíngueZ, R. J. (1846), Diccionario nacional, Madrid, Miguel Guijarro.

Esqué, M. D. y CARMONA, A. M. (1995a), «La biblioteca de Martí i Franquès», en PuIGPLA, C. et alii (eds.), Actes de les III trobades d'història de la ciència i de la tècnica als Països Catalans, Barcelona, SCHCT, pp. 397-402.

Esqué, M. D. y CARMONA, A. M. (1995b), «Aportació dels insvestigadors de la història de la ciència als Països Catalans al coneixement de la figura d'Antoni Martí i Franquès», En Puig-PlA, C. et alii (eds.), Actes de les III trobades d'història de la ciència $i$ de la tècnica als Països Catalans, Barcelona, SCHCT, pp. 391-393.

Gago, R. (1978), «Bicentenario de la fundación de la Cátedra Química de Vergara. El proceso de constitución», Llull, 2, pp. 5-18.

Gago, R. (1982), «Introducción», en LAvoisier, A. L., Tratado elemental de química, Madrid, Alfaguara, XIII-LXVIII

GAgO, R. (1988a), «The New Chemistry in Spain», Osiris, 4, pp. 169-192.

GAGO, R. (1988b), «Cultivo y enseñanza de la química en la España de principios del siglo XIX», en SÁnchez Ron, J. M. (ed.), Ciencia y sociedad en España, de la Ilustración a la Guerra Civil, Madrid, El Arquero, pp. 129-142.

Gago, R. (1990), «Luis Proust y la cátedra de química de la Academia de Artillería de Segovia», en Proust, L., Anales del Real Laboratorio de Química de Segovia, Segovia, A. Espinosa [1795]; facsímil en Segovia, Academia de Artillería, pp. 5-51.

GAGO, R. y CARRILlO, J. L. (1979), La introducción de la nueva nomenclatura y el rechazo de la teoría de la acidez de Lavoisier, Málaga, Universidad. 
Gago, R. y Pellón, I. (1994), Historia de las Cátedras de Química y Mineralogía de Bergara a finales del siglo XVIII. Bergara, Ayuntamiento.

GÁllego, R. (2002), «Notas sobre la historia del léxico de la fotografía en español», en ECHENIQUe, M. T. y SÁNCHEZ, J. (eds.), Actas del V Congreso internacional de historia de la lengua española, Madrid, Gredos, pp. 2051-2062.

García Belmar, A. y Bertomeu SÁnchez, J. R. (1999), Nombrar la materia. Una introducción histórica a la terminología química, Barcelona, Serbal.

García Belmar, A. y Bertomeu SÁnchez, J. R. (2001), «Viajes a Francia para el estudio de la química, 1770 y 1833», Asclepio, LIII-1, pp. 95-140.

GARRIGA, C. (1996), «Apuntes sobre la incorporación del léxico de la química al español, la influencia de Lavoisier», Documents pour l'histoire du français langue étrangère ou seconde, 18, pp. 419-435.

GARRIGA, C. (1996-97), «Penetración del léxico químico en el DRAE, la edición de 1817», Revista de Lexicografía, 3, pp. 59-80.

GARRIGA, C. (1997), «La recepción de la Nueva nomenclatura química en español», Grenzgänge, 8, pp. 33-48.

GARRIGA, C. (1998a), «Química, enseñanza y divulgación de la terminología, las Lecciones de química teórica y práctica de Morveau, Maret y Durande», en BRUMME, J. (ed.), La historia de los lenguajes iberorrománicos de especialidad (siglos XVII-XIX); soluciones para el presente, Barcelona, UPF, pp. 163-174.

GARRIGA, C. (1998b), «Luis Proust y la consolidadción de la terminología química en español», en GarcíA, J. L.; Moreno, J. M.; RuIZ, G. (eds.), VI Congreso de la Sociedad Española de Historia de las Ciencias y de las Técnicas, Segovia, Junta de Castilla y León, pp. 691-699.

GARriga, C. (1998c), «El Diccionario Universal de Física de Brisson (1796-1802) y la fijación lexicográfica de la terminología química en español», en GARCÍA TURZA, C.; GonZÁlez, F.; MAngado, J. (eds.), Actas del IV Congreso Internacional de Historia de la Lengua Española, Logroño, AHLE - Gobierno de La Rioja - Universidad de La Rioja, pp. 179-190.

GARRIGA, C. (2001a), «Sobre el diccionario académico, la 12a ed. (1884)», en MEDINA GuERrA, A. M. (ed.), Estudios de lexicografía diacrónica del español, Málaga, Universidad, pp. 261-315.

GARRIGA, C. (2001b), «Notas sobre el vocabulario de la química orgánica en español, Liebig y la divulgación de los derivados en -ina», en BARGALlÓ, M.; FORGAS, E.; GARriga, C.; Schnitzer, J.; RubiO, A. (eds.), Las lenguas de especialidad y su didáctica, Tarragona, Universitat Rovira i Virgili, pp. 169-180.

GARRIGA, C. (2002a), «Notas sobre la incorporación de los términos de elementos químicos en español en el s. XIX, el Léxico histórico y sinonímico de Ferdinand Hoefer», en PöLl, B. y RAINER, F. (eds.), Vocabula et vocabularia. Études de lexicologie et de (méta-)lexicographie romanes en l'honneur du 60e anniversaire de Dieter Messner, Frankfurt am Mein, Peter Lang, pp. 131-144. 
GARRIGA, C. (2002b), «Notas sobre la incorporación del sufijo técnico -ona al español», en ECHENIQUe, M. T. et al. (eds.), Actas del V Congreso Internacional de Historia de la Lengua Española, Madrid, Gredos, pp. 2093-2106.

GARRIGA, C. (2003), «Aspectos de la adaptación de la nueva nomenclatura química al español», GUTIÉRrez, B. (ed.), Aproximaciones al lenguaje de la ciencia, Salamanca, Universidad, en prensa.

Garriga, C.; AngladA, E.; Bajo, E.; Madrona, A. y SAla, L. (2001), «Proyecto, la formación de la terminología química en español», en BRUMME, J. (ed.), La historia de los lenguajes iberorrománicos de especialidad, la divulgación de la ciencia, Barcelona - Frankfort del Meno - Madrid, Universitat Pompeu Fabra - Vervuert - Iberoamericana, pp. 105-117.

GARrigós, Ll. (1990), Contribución al estudio de la constitución del lenguaje químico en castellano, Valencia, Universidad (edición en microficha).

GÓMEZ DE ENTERRÍA, J. (1998), «Consideraciones sobre la terminología científicotécnica de carácter patrimonial en el español del siglo XVIII», Boletín de la Real Academia Española, 78, pp. 275-301.

GutiÉRrez CuAdrado J. (1994), «El Diccionario Hispano-americano de Montaner y Simón», Actas del XXIX Congreso del Instituto Internacional de Literatura Iberoamericana, Barcelona, Universidad, pp. 263-282.

GutiÉRREZ CUADRADO, J. (1998a), «Torres Muñoz de Luna y la lengua de la química en el siglo XIX», en GARCÍA, J. L.; MorenO, J. M.; RUIZ, G. (eds.), VI Congreso de la Sociedad Española de Historia de las Ciencias y de las Técnicas, Segovia, Junta de Castilla y León, pp. 701-711.

Gutiérrez CUADrado, J. (1998b), «F. Carbonell y Bravo y su texto Curso analítico de química escrito en italiano por F. Mojón», en GARCÍA TURZA, C.; GONZÁLEZ, F.; MANGADO, J. (eds.), Actas del IV Congreso Internacional de Historia de la Lengua Española, Logroño, AHLE - Gobierno de La Rioja - Universidad de La Rioja, pp. 219-230.

GutiÉRreZ CUADRADO, J. (2001), «Lengua y ciencia en el siglo XIX español, el ejemplo de la química», en Bargalló, M., Forgas, E., Garriga, C., Rubio, A. y SchnitZER, J. (eds.), Las lenguas de especialidad y su didáctica, Tarragona, Universitat Rovira i Virgili, pp. 181-196.

Gutiérrez CuAdrado, J. y PAscual J. A. (1992), «Prólogo a propósito de las Actas del Congreso Literario Hispano-americano de 1892», en ASOCIACIÓN DE ESCRITORES Y ARTistas EsPañoles (1893), Congreso Literario Hispano-americano, Madrid, Ricardo Fé (edición facsímil en Madrid, Instituto Cervantes - Pabellón de España - Biblioteca Nacional, 1992), pp. IX-XXXI.

HoEfer, F. (1845), Nomenclature et classifications chimiques (...). Paris, Baillière.

HoEFER, F. (1853), Nomenclatura y clasificaciones químicas (...). Madrid, M. A. Gil.

IGLESIA, S. (2002), Las voces de la química en el Diccionario Nacional de R. J. Domínguez, la marcación y la definición, Barcelona, Universitat Pompeu Fabra (trabajo de investigación). 
LAVOISIER, A. L. (1798), Tratado elemental de química, Madrid, Imprenta Real.

LÁZARO CARRETER, F. (1985), Las ideas lingüísticas en España durante el siglo XVIII, Barcelona, Crítica.

LiEBIG, J. (1847-48), Tratado de química orgánica, Madrid, La Ilustración.

LÓPEZ PIÑERO, J. M. (1982a), «La literatura científica en la España contemporánea», en DíAz PlajA, G. (dir.), Historia general de las literaturas hispánicas (vol. VI), Barcelona, Vergara, pp. 675-693.

LÓPEZ PIÑERO, J. M. (1982b), La ciencia en la historia hispánica, Barcelona, Salvat.

LÓPEZ PIÑERO, J. L. (1992), «Introducción», en LÓPEZ PIÑERO, J. L. (ed.), La ciencia en la España del siglo XIX, Madrid, Marcial Pons, pp. 11-18.

LóPeZ Piñero, J. Ma ; Glick, T.; NAvarro, V.; y Portela, E. (1983), Diccionario histórico de la ciencia moderna en España, Barcelona, Península.

MARTÍ I FRANQUÈS, A. (1787), «Sobre algunas producciones que resultan de la combinación de varias sustancias aeriformes», en QuINTANA, A. (1935), Antoni de Martí $i$ Franquès; memòries originals, estudi biogràfic $i$ documental, Barcelona, Acadèmia de Ciències i Arts, pp. 11-20.

Morveau, L.; Lavoisier, A. L.; Berthollet, C. y Fourcroy, F. (1787), Méthode de nomenclature chimique, París, Cuchet.

Morveau, L.; Lavoisier, A. L.; Berthollet, C. y Fourcroy, F. (1788), Método de la nueva nomenclatura química, Madrid, Antonio de Sancha.

MoYA, T. (1994), «La enseñanza de la química en la universidad española del siglo XIX», en Asclepio, XLVI-2, pp. 43-57.

NiETo, A. (1996), «Martí i Franquès, Carbonell i Bravo, i els usos de la nova química a la Catalunya il.lustrada», en IZQUIERDO, M. et alii (eds.), Lavoisier i els orígens de la química moderna, 200 anys després (1794-1994), Barcelona, SCHCYT, pp. 159-184.

PeSET, J. L. (1988), «Educación y ciencia en el fin del Antiguo Régimen», en SÁNCHEZ Ron, J. M. (ed.), Ciencia y sociedad en España, de la Ilustración a la Guerra Civil, Madrid, El Arquero, pp. 17-25.

Peset, J. L. y LAFUente, A. (1988), «Las actividades e instituciones científicas en las España ilustrada», en Sellés, M.; Peset, J. L. y LAfuente, A. (eds.), Carlos III y la ciencia de la Ilustración, Madrid, Alianza, pp. 29-79.

PESET, M. y PESET, J. L. (1974), La universidad española (siglos XVIII-XIX), Madrid, Taurus.

Peset, M. y Peset, J. L. (1992), «Las universidades españolas del siglo XIX y las ciencias», en LÓPEZ PIÑERO, J. L. (ed.), La ciencia en la España del siglo XIX, Madrid, Marcial Pons, pp. 19-49.

PORTElA, E. (1998), La química en el siglo XIX, Madrid, Akal.

PORTElA, E. (1999), La química ilustrada, Madrid, Akal. 
Portela, E. y Soler, A. (1992), «La química española en el siglo XIX», en LÓPEZ PIÑERO, J. M. (ed.), La ciencia en la España del siglo XIX, Madrid, Marcial Pons, pp. 85-107.

Proust, L. (1791), Anales del Real Laboratorio de Química de Segovia, Segovia, A. Espinosa; ed. facsímil de R. Gago, Segovia, Academia de Artillería, 1990.

R.A.E. (1726-1739), Diccionario de la lengua castellana, Madrid, Francisco del Hierro; facsímil en Madrid, Gredos, 1984.

R.A.E. (1780), Diccionario de la lengua castellana, Madrid, J. Ibarra; facsímil en Madrid, Espasa-Calpe.

R.A.E. (1783), Diccionario de la lengua castellana, Madrid, J. Ibarra, $2^{\mathrm{a}}$ ed.

R.A.E. (1791), Diccionario de la lengua castellana, Madrid, Vda. de Ibarra, $3^{\mathrm{a}}$ ed. R.A.E. (1803), Diccionario de la lengua castellana, Madrid, Vda. de Ibarra, $4^{\mathrm{a}}$ ed.

R.A.E. (1817), Diccionario de la lengua castellana, Madrid, Imprenta Real, $5^{\mathrm{a}}$ ed.

R.A.E. (1837), Diccionario de la lengua castellana, Madrid, Imprenta Nacional, 8 a ed.

R.A.E. (1869), Diccionario de la lengua castellana, Madrid, M. Rivadeneyra, $11^{\mathrm{a}} \mathrm{ed}$.

R.A.E. (1884), Diccionario de la lengua castellana, Madrid, Gregorio Hernando, $12^{\mathrm{a}}$ ed.

R.A.E. (1899), Diccionario de la lengua castellana, Madrid, Hernendo y compañía, $13^{\mathrm{a}}$ ed.

R.A.E. (1925), Diccionario de la lengua española, Madrid, Espasa-Calpe, $15^{\mathrm{a}} \mathrm{ed}$.

R.A.E. (2001), Diccionario de la lengua española, Madrid, Espasa-Calpe, $22^{\mathrm{a}} \mathrm{ed}$.

RAINER, F. (1993), Spanische Wortbildungslehre, Tübingen, Niemeyer.

RoIG, C. (1995), «El Nuevo diccionario francés-español de Antonio de Capmany», en LAFARGA y otros (eds.), La traducción. Metodología-historia-literatura. Ámbito hispanofrancés. Barcelona, PPU, pp. 75-80.

SARRAILH, J. (1985), La España Ilustrada de la segunda mitad del siglo XVIII, Madrid, FCE.

SECO, M. (1987a), «El nacimiento de la lexicografía moderna no académica», en Estudios de lexicografia española, Madrid, Paraninfo, pp. 129-151,

SECO, M. (1987b), «Ramón Joaquín Domínguez», en Estudios de lexicografía española, Madrid, Paraninfo, pp. 152-164.

SECO, M. (1991), «Introducción», en R.A.E (1780), Diccionario de la lengua castellana, Madrid, J. Ibarra; facsímil en Madrid, Espasa-Calpe, pp. III-XII.

TERreros, E. (1786), Diccionario castellano con las voces de ciencias y artes, Madrid, Vda. de Ibarra; facsímil en Madrid, Arco-Libros, 1987. 